\title{
Electrorotation of arbitrarily shaped micro-objects: modeling and experiments
}

\author{
Tomáš Michálek, Aude Bolopion, Zdeněk Hurák, and Michaël Gauthier
}

\begin{abstract}
In this paper, we study the electrorotational behavior of non-spherical micro-objects. We extend a controloriented model of general dielectrophoresis to incorporate also the hydrodynamics so that we can predict the motion of nonspherical micro-objects in fluidic environments. Such mathematical (computational) model enables model-based feedback control of a position and orientation of particles by real-time (online) computation of voltages applied to the electrodes. We use the measured data from experiments with electrorotation of an artificial micro-object having a Tetris-like shape, to evaluate the performance of the proposed model. We also demonstrate the qualitative difference in behavior from the commonly performed electrorotation of a sphere advocating the necessity for modelbased control. Further analysis of the simulation results for other than the experimentally explored scenarios provides additional useful insight into the electrorotational behavior of non-spherical objects.
\end{abstract}

Index Terms-electrorotation, non-spherical objects, controloriented model

\section{INTRODUCTION}

$\mathbf{E}$ LECTROROTATION (ROT), as one of the AC electrokinetic effects, provides a way to induce a rotational motion of micro-objects by an external electric field in a non-contact fashion. The background mechanism of ROT is closely related to the mechanisms of electro-orientation (EO) or dielectrophoresis (DEP) forming a family of phenomena also called as generalized dielectrophoresis (gDEP). First description of these phenomena were provided by Pohl in [1] and a subsequent detailed theoretical background of all the mentioned gDEP effects can be found for example in [2]-[5], short summary in [6] and a review of gDEP applications in [7].

Mostly, ROT was used in an open-loop regime for characterization of micro-objects (often biological cells) through measurements of their so-called electrorotational spectra [8][10]. In [11], a feedback system was proposed for a stochastic estimation of dielectric properties of spherical particles.

ROT can be, however, used also for a closed-loop feedback orientation control of micro-objects, similarly as the DEP was already used for their position control [12]-[14]. Non-contact manipulation, including both positioning and orientation, is a sought skill for example in biology or micro-assembly

T. Michálek and Z. Hurák were with the Faculty of Electrical Engineering, Department of Control Engineering, Czech Technical University in Prague, Karlovo namesti 13, 121 35, Prague, Czech Republic e-mail: tomas.michalek@fel.cvut.cz.

A. Bolopion and M. Gauthier are with FEMTO-ST Institute, AS2M department Univ. Bourgogne Franche-Comté, CNRS, 24 rue Savary, F-25000 Besançon, France email: aude.bolopion@femto-st.fr.

Manuscript received March 25, 2019.

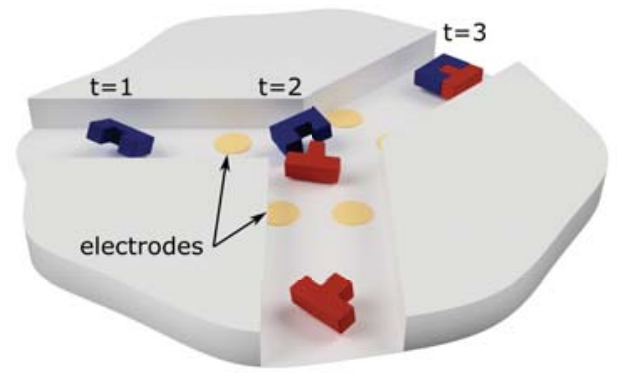

Fig. 1. Foreseen application of gDEP-micro-electrodes at the junction of two microfluidic channels generate controlled electric field for non-contact assembly of micro-objects into the desired products.

application domains. In [15], Jiang et al. demonstrated the feedback orientation control on a case of spherical yeast cells. Most of the objects of interest in the mentioned application domains are, however, often non-spherical (e.g., red blood cells or virus bodies in biology or completely artificial shapes in micro-assembly). An example task solved in the field of micro-assembly is shown in Fig. 1. Consider a device formed by two microfluidic channels merging in a junction. Through each of these two channels, objects of two distinct shapes are conveyed to the junction where a set of microelectrodes can dielectrophoretically actuate them. Here, ROT could be used to orient suitably both of the objects, one w.r.t. to the other, and DEP would simultaneously bring them near to each other so that they assemble. The finished product would then continue along the flow through the output channel.

Although the use of spheres, as the manipulated objects, is quite common in DEP and ROT experiments, when it comes to non-spherical objects the results are mostly limited to less or more prolate ellipsoids. Miller and Jones [16] used EO to characterize the electrical properties (internal conductance and permittivity) of human and llama erythrocytes. In [17], EO was used for characterization of microtubules. Both ROT and EO were used in [18] for characterization of surface conductance and permittivity of artificial SU-8 micro-rods with variously functionalized surfaces. In all of these works, the objects were approximated for analysis purposes as (possibly layered) ellipsoids. In [19], Egger, et al. derived a model for ROT of dumb-bell shaped objects (two connected spheres) and applied it in their experiments with single erythrocytes and pollen. Arcenegui et al. presented in [20] a theory for EO and ROT of slender cylindrical objects and validated it in experiments with metal nanowires. Both positive and negative 
DEP manipulation of rod-shaped viruses were demonstrated in [21]. A quantitative agreement with an expression for DEP force acting on a homogeneous dielectric cylinder was obtained. Apart from the experimental results, there exist several purely numerical simulation studies [22], [23].

Except for the missing theory and experiments involving objects of shapes other than spheres, ellipsoids or cylinders (often modeled as ellipsoids), it is also the hydrodynamics governing the interaction of these objects with the surrounding fluidic medium, which is usually left without discussion in gDEP experiments. Mathai et al., in their work [24] on position and orientation control using electroosmotic flows, dealt with modeling of hydrodynamic forces and torques influencing the motion of ellipsoidal objects. A complete and exhaustive treatment of hydrodynamics at low Reynolds number are given by Happel and Brenner in [25].

\section{Contribution}

In this paper, we couple our recently published controloriented model of DEP for arbitrarily-shaped objects [26] with control-oriented model of hydrodynamics working again with arbitrarily-shaped objects based on [25]. The resulting model then allows us to predict both the translational and rotational velocities of the micro-object of interest and thus simulate its motion in a fluidic environment. Such model, evaluable in real-time (in fractions of a second), is motivated by and can be used for design and implementation of model-based control algorithms (e.g., for micro-assembly applications).

We demonstrate the performance of the model by comparing its outcomes against experimental observations. We perform an electrorotation with Tetris-shaped micro-object as a task in which both gDEP and hydrodynamics play a crucial role.

\section{MATHEMATICAL MODEL}

The purpose of the mathematical model is to predict the motion of an arbitrarily shaped micro-object in a fluidic medium under the influence of an external electric field generated by a set of microelectrodes. Our ultimate goal is to make the model a part of a control algorithm that sets the voltages applied to the electrodes so that an object follows a planned trajectory. To this purpose, we require that the model be evaluable in real-time (in fractions of a second) similarly as in [26]. Despite its simplicity, which is necessary for its fast evaluation, such a model should still take into account all the relevant physical phenomena for accurate enough results. In our case, the electrokinetics and hydrodynamics represent the dominant effects, and we will further deal with them in the following subsections.

We will neglect the other, unobserved or not so influential, effects to keep the model fast to evaluate. A feedback controller will then compensate for the discrepancies between the model and the reality. These neglected effects include, for example, the thermally induced fluid flows or friction forces between the object and other solid parts of the experimental chamber.

\section{A. AC electrokinetics}

For actuation, we drive the micro-electrodes with harmonic signals having a frequency of $25 \mathrm{kHz}$, which is high enough to allow us to consider just the gDEP forces and torques and to neglect all the other effects like electrolysis, electroosmosis, etc [27]. We further decided to ignore any electrical interaction of the object with the walls or the bottom floor of the experimental chamber, since a sufficiently large distance separates them during the experiments.

The critical enabling principle used in gDEP is the electric polarization of the object of interest, which takes place when it is exposed to an external electric field. From the various polarization mechanisms (electronic, atomic, orientational, etc.), it is the interfacial polarization caused by a longrange charge transport in dielectrics, whose effect prevails in the AC electrokinetics applications (micro-objects located in fluidic mediums). The polarization is not instantaneous, but the charge rather gradually builds up at the outer as well as the inner interfaces of the object with speed characterized by a relaxation time $\tau$ (a period, in which the maximum polarization takes place).

Thus the polarization depends on the frequency of the external AC electric field. With frequencies sufficiently below $f_{\text {co }}=1 /(2 \pi \tau)$, the charges manage to follow the changing field whereas with frequencies sufficiently above this threshold, they will not be able to 'catch up' the field at all and no polarization will take place. The frequency response is strongly influenced by the material properties of the object and the medium. At lower frequencies, these are the conductivities, which have the major influence over the polarizability. At higher frequencies, the impact of permittivity takes over. All of the above characteristics are captured in the so-called ClausiusMossotti factor [6].

The resulting charge structure emerging at the interface of the object then interacts through Coulomb forces with the original electric field exerting forces and torques on the object itself. Based on whether the object is more polarizable than the medium (at the given frequency) or vice versa, it will be attracted to (so-called positive DEP) or repelled from (socalled negative DEP), respectively, the places of high electric field intensity. A non-spherical object will tend to orient along the electric field lines. In case of a rotating electric field that continuously changes its orientation, the object will keep rotating as the charge distribution on its interface attempts to 'catch up' with the field (so-called cofield rotation) or keeps being pushed ahead of the field (so-called antifield rotation) as repelled by the charges on the electrodes. These phenomena are, in the given order, the already mentioned DEP, EO, and ROT.

To compute the gDEP forces and torques, we use our recently published model described in [26], which, unlike others, can also deal with non-spherical objects. Precomputing a "basis table" for the given specific shape of the object, it then provides a way for calculation of its multipolar representation in real time (in fractions of a second). As a consequence, also the gDEP force and torque can be computed this fast. An implementation of the described model is freely available at 
https://goo.gl/eGZY7P.

\section{B. Hydrodynamics}

Except for the electrokinetic forces, the other significant forces and torques that act on the object are the ones caused by hydrodynamics. As the objects moves through and revolves inside the fluidic medium, it will experience hydrodynamic drag force and torque. They not only oppose the object's motion slowing down its translation or rotation but in case of a non-orthotropic object (it does not have three perpendicular planes of symmetry), they also couple the translational and rotational motion. From a merely translational movement at the beginning, a hydrodynamic drag torque can emerge forcing the object to rotate or vice versa- a rotational motion can propel the object throughout the fluid. This effect is the basic locomotion principle of the robotic microswimmers [28], [29].

The nonlinear partial differential Navier-Stokes equations governing the fluid flow are generally hard to solve. Timeand memory-demanding finite element method (FEM) has to be usually deployed. Since we aim at constructing a control-oriented model evaluable in real time, we pose a few simplifying assumptions valid for microfluidic systems. For low Reynolds numbers (small dimensions and low velocities typical for microfluidic systems) and incompressible fluids of constant viscosity $\mu$, the governing Navier-Stokes equations simplify to the so-called Stokes flow (or creeping flow) equations [25]

$$
\begin{aligned}
\nabla^{2} \boldsymbol{v} & =\frac{1}{\mu} \nabla p, \\
\nabla \cdot \boldsymbol{v} & =0 .
\end{aligned}
$$

Here, $\boldsymbol{v}$ is the vector field representing fluid velocity, $p$ is the scalar pressure field, and $\nabla$ is the vector differential operator. Furthermore, to simplify the explanation, we first consider the fluid to be unbounded. This is reasonable if the object does not take place in close vicinity of the bottom of the chamber or its walls. We will revisit this assumption at the end of this section.

Although the Stokes flow equations are generally solvable just by using FEM, they are at least linear. Omitting the details discussed in [25], this allows us to formulate the model for hydrodynamic drag force, $\mathbf{F}$, and torque $\mathbf{T}_{O}$ around a point $O$ inside the object as

$$
\begin{aligned}
& \mathbf{F}_{\text {drag }}=-\mu \mathbf{K} \mathbf{v}-\mu \mathbf{C}_{O}^{\mathrm{T}} \boldsymbol{\omega}, \\
& \mathbf{T}_{\text {drag }}=-\mu \mathbf{C}_{O} \mathbf{v}-\mu \boldsymbol{\Omega}_{O} \boldsymbol{\omega} .
\end{aligned}
$$

Here, $\mathbf{v}$ is the translational velocity of the object, $\boldsymbol{\omega}$ is its angular speed and $\mathbf{K}, \mathbf{C}_{O}$, and $\boldsymbol{\Omega}_{O}$ are translational, coupling and rotational tensors, respectively. The choice of $O$ is arbitrary, but if it is the center of the hydrodynamic reaction, (2) poses the most symmetric form $\left(\mathbf{C}_{O}\right.$ is then symmetric). We chose $O$ to be at the geometric center of the object.

These tensors contain all the shape-specific information and can be computed beforehand using FEM just once for each specific shape of the object. We do so by solving (1) for six different sets of boundary conditions. The first three of them are

$$
\boldsymbol{v}=\mathbf{U}_{i} \text { on } \mathcal{S}_{p}, i=1,2,3,
$$

where $\boldsymbol{v} \rightarrow 0$ as $\boldsymbol{r}_{O} \rightarrow \infty, \mathcal{S}_{p}$ denotes the surface of the object and $\boldsymbol{r}_{O}$ is a position vector having an origin at $O$. We set $\mathbf{U}_{1}=[1,0,0]^{\mathrm{T}}, \mathbf{U}_{2}=[0,1,0]^{\mathrm{T}}$ and $\mathbf{U}_{3}=[0,0,1]^{\mathrm{T}}$ so that they correspond to translational movements of the object in a quiescent fluid along the three coordinate axes. The remaining three boundary conditions are then

$$
\boldsymbol{v}=\boldsymbol{\omega}_{i} \times \boldsymbol{r}_{O} \text { on } \mathcal{S}_{p}, i=1,2,3,
$$

with $\boldsymbol{v} \rightarrow 0$ as $\boldsymbol{r}_{O} \rightarrow \infty$, and vector of angular velocities set as $\boldsymbol{\omega}_{1}=[1,0,0]^{\mathrm{T}}, \boldsymbol{\omega}_{2}=[0,1,0]^{\mathrm{T}}$ and $\boldsymbol{\omega}_{3}=[0,0,1]^{\mathrm{T}}$ corresponding to rotation of the object in a quiescent fluid about the three coordinate axes. The total computation time was under 30 min using a regular PC. We denote the individual solutions in the order in which the distinct boundary conditions were given as velocity and pressure fields $\boldsymbol{v}^{i}$ and $p^{i}, i=$ $1,2, \ldots, 6$, respectively. Based on these, we then compute the position dependent quantities

$$
\begin{aligned}
\mathbf{P}_{1}^{i}= & {\left[\begin{array}{c}
-\frac{p^{i}}{\mu}+2 \frac{\partial v_{x}^{i}}{\partial x} \\
\frac{\partial v_{y}^{i}}{\partial x}+\frac{\partial v_{x}^{i}}{\partial y} \\
\frac{\partial v_{z}^{i}}{\partial x}+\frac{\partial v_{x}^{i}}{\partial z}
\end{array}\right], \mathbf{P}_{2}^{i}=\left[\begin{array}{c}
\frac{\partial v_{x}^{i}}{\partial y}+\frac{\partial v_{y}^{i}}{\partial x} \\
-\frac{p^{i}}{\mu}+2 \frac{\partial v_{y}^{i}}{\partial y} \\
\frac{\partial v_{z}^{i}}{\partial y}+\frac{\partial v_{y}^{i}}{\partial z}
\end{array}\right], } \\
\mathbf{P}_{3}^{i}= & {\left[\begin{array}{c}
\frac{\partial v_{x}^{i}}{\partial z}+\frac{\partial v_{z}^{i}}{\partial x} \\
\frac{\partial v_{y}^{i}}{\partial z}+\frac{\partial v_{z}^{i}}{\partial y} \\
-\frac{p^{i}}{\mu}+2 \frac{\partial v_{z}^{i}}{\partial z}
\end{array}\right] . }
\end{aligned}
$$

The desired tensors $\mathbf{K}, \mathbf{C}_{O}$, and $\boldsymbol{\Omega}_{O}$ are then obtained as

$$
\begin{aligned}
& \mathbf{K}=\left[\mathbf{K}_{1}, \mathbf{K}_{2}, \mathbf{K}_{3}\right], \\
& \quad \text { with } \mathbf{K}_{i}=-\iint_{\mathcal{S}_{p}}\left[\mathbf{P}_{1}^{i}, \mathbf{P}_{2}^{i}, \mathbf{P}_{3}^{i}\right] \mathrm{d} \mathbf{S}, \\
& \mathbf{C}_{O}=\left[\mathbf{C}_{1}, \mathbf{C}_{2}, \mathbf{C}_{3}\right], \\
& \quad \text { with } \mathbf{C}_{i}=-\iint_{\mathcal{S}_{p}}\left[\boldsymbol{r}_{O} \times \mathbf{P}_{1}^{i}, \boldsymbol{r}_{O} \times \mathbf{P}_{2}^{i}, \boldsymbol{r}_{O} \times \mathbf{P}_{3}^{i}\right] \mathrm{d} \mathbf{S}, \\
& \boldsymbol{\Omega}_{O}=\left[\boldsymbol{\Omega}_{4}, \boldsymbol{\Omega}_{5}, \boldsymbol{\Omega}_{6}\right], \\
& \quad \text { with } \boldsymbol{\Omega}_{i}=-\iint_{\mathcal{S}_{p}}\left[\boldsymbol{r}_{O} \times \mathbf{P}_{1}^{i}, \boldsymbol{r}_{O} \times \mathbf{P}_{2}^{i}, \boldsymbol{r}_{O} \times \mathbf{P}_{3}^{i}\right] \mathrm{d} \mathbf{S} .
\end{aligned}
$$

Please notice that although the expressions for $\mathbf{C}_{i}$ and $\boldsymbol{\Omega}_{i}$ are the same, the tensors $\mathbf{C}_{O}$ and $\boldsymbol{\Omega}_{O}$ will differ, because different values of indices $i$ (and thus data from differing simulations) are used for their computation.

For cases when the object moves in the vicinity of solid obstacles (e.g., walls or bottom of the experimental chamber), we can not consider the fluid to be unbounded. In such situations, tensors $\mathbf{K}, \mathbf{C}_{O}$, and $\boldsymbol{\Omega}_{O}$ need to be computed repeatedly for various positions of the object to the walls and stored in a lookup table from where they can be accessed and interpolated in real time.

Our numerical simulations for the Tetris shape revealed that below $\sim 130 \mu \mathrm{m}$ the error in drag torque if we use the unbounded fluid assumption, is larger than $1 \%$. Therefore we utilized the described approach to take into account the 
proximity of the bottom floor of the chamber when comparing the simulations to experimental behavior in section $\mathrm{V}$.

\section{Resulting model}

Now, that we know both the propulsion and frictional forces and torques caused by the electrokinetics and hydrodynamics, respectively, we can state the general model describing the motion of the object

$$
\begin{aligned}
\dot{\boldsymbol{x}} & =\boldsymbol{v}_{\mathrm{O}} \\
\dot{\boldsymbol{v}}_{\mathrm{O}} & =\frac{1}{m} \mathbf{F}_{\mathrm{DEP}}+\frac{1}{m} \mathbf{F}_{\mathrm{sed}}-\frac{1}{m} \mathbf{F}_{\mathrm{drag}} \\
\dot{\boldsymbol{\theta}} & =\boldsymbol{\omega} \\
\dot{\boldsymbol{\omega}} & =\mathbf{I}^{-1} \mathbf{T}_{\mathrm{DEP}}-\mathbf{I}^{-1} \mathbf{T}_{\mathrm{drag}},
\end{aligned}
$$

where $\boldsymbol{x}=[x, y, z]^{\mathrm{T}}$ is a vector representing the position of the center of the object, $\boldsymbol{v}_{\mathrm{o}}=\left[v_{\mathrm{x}}, v_{\mathrm{y}}, v_{\mathrm{z}}\right]^{\mathrm{T}}$ is its translational velocity, $\mathbf{F}_{\mathrm{DEP}}=\left[F_{\mathrm{DEP}, \mathrm{x}}, F_{\mathrm{DEP}, \mathrm{y}}, F_{\mathrm{DEP}, \mathrm{z}}\right]^{\mathrm{T}}$ is the DEP force, $\mathbf{F}_{\text {sed }}=\left[0,0,\left(\rho_{\text {medium }}-\rho_{\text {object }}\right) V g\right]^{\mathrm{T}}$ is the sedimentation force (buyonacy and gravity effect) and $\mathbf{F}_{\text {drag }}=\left[F_{\text {drag, } \mathrm{x}}, F_{\text {drag,y }}, F_{\text {drag, } \mathrm{z}}\right]^{\mathrm{T}}$ is the hydrodynamic drag force. Similarly, $\dot{\boldsymbol{\theta}}=[\psi, \theta, \phi]^{\mathrm{T}}$ is a vector comprised of Euler angles representing the orientation of the object in space (successive rotations about the $\mathrm{x}-, \mathrm{y}-$ and $\mathrm{z}$-axes of the coordinate frame), $\boldsymbol{\omega}=\left[\omega_{\mathrm{x}}, \omega_{\mathrm{y}}, \omega_{\mathrm{z}}\right]^{\mathrm{T}}$ is then the rotational velocity and $\mathbf{T}_{\mathrm{DEP}}$ and $\mathbf{T}_{\mathrm{drag}}$ are the DEP and hydrodynamic drag torques, respectively. The mass of the object is $m=V \rho_{\text {object }}$, with $V$ being the object's volume and $\rho_{\text {object }}$ its density. Finally, $\rho_{\text {medium }}$ is the density of the fluidic medium, $g$ is the acceleration due to the gravity and $\mathbf{I}$ is the object's moment of inertia.

In practice, however, the inertia of the object is negligible due to its low weight, and the object achieves its final translational and rotational velocity immediately without any noticeable transients. Analogously, the object immediately stops moving or rotating due to the DEP when the voltage applied to the electrodes is suddenly switched off. The general model represented by (6) can therefore be simplified using

$$
\begin{aligned}
\dot{\boldsymbol{v}}_{\mathrm{O}} & =0 \\
\dot{\boldsymbol{\omega}} & =0,
\end{aligned}
$$

from which the steady state values of velocities follow and the new motion equations are

$$
\begin{gathered}
\dot{\boldsymbol{x}}=\boldsymbol{v}_{\text {o,final }} \\
\dot{\boldsymbol{\theta}}=\boldsymbol{\omega}_{\text {final }},
\end{gathered}
$$

where $\mathbf{v}_{\text {final }}$ and $\boldsymbol{\omega}_{\text {final }}$ are the mentioned final translational and rotational velocities occurring when all the forces and torques are balanced. This amounts to solving the following linear system of equations based on (2)

$$
-\mu\left[\begin{array}{cc}
\mathbf{K} & \mathbf{C}_{O}^{\mathrm{T}} \\
\mathbf{C}_{O} & \boldsymbol{\Omega}_{O}
\end{array}\right]\left[\begin{array}{c}
\boldsymbol{v}_{\mathrm{o}, \text { final }} \\
\boldsymbol{\omega}_{\text {final }}
\end{array}\right]=\left[\begin{array}{c}
\mathbf{F}_{\text {drag }} \\
\mathbf{T}_{\text {drag }}
\end{array}\right]=-\left[\begin{array}{c}
\mathbf{F}_{\text {DEP }}+\mathbf{F}_{\text {sed }} \\
\mathbf{T}_{\text {DEP }}
\end{array}\right],
$$

which leads us to

$$
\left[\begin{array}{c}
\dot{\boldsymbol{x}} \\
\dot{\boldsymbol{\theta}}
\end{array}\right]=\frac{1}{\mu}\left[\begin{array}{cc}
\mathbf{K} & \mathbf{C}_{O}^{\mathrm{T}} \\
\mathbf{C}_{O} & \boldsymbol{\Omega}_{O}
\end{array}\right]^{-1}\left[\begin{array}{c}
\mathbf{F}_{\mathrm{DEP}}+\mathbf{F}_{\mathrm{sed}} \\
\mathbf{T}_{\mathrm{DEP}}
\end{array}\right] .
$$

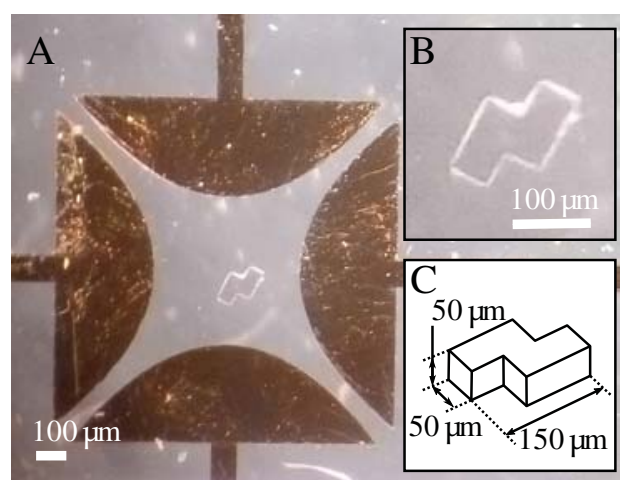

Fig. 2. Electrode array and the micro-object having a shape of one of the Tetris pieces.

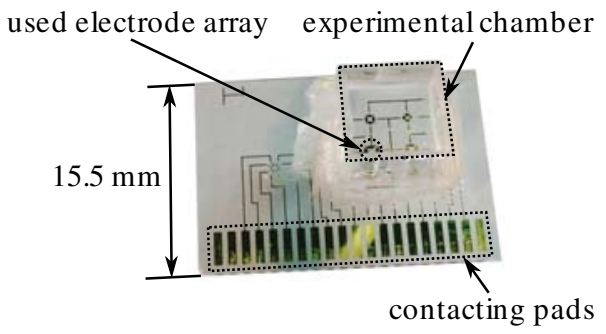

Fig. 3. Experimental glass chip with an electrode array and a plastic basin.

\section{DESCRIPTION OF THE USED EXPERIMENTAL SETUP}

\section{A. Micromanipulation workplace}

To induce DEP motion, we used a quadrupolar electrode array shown in Fig. 2A, which is commonly used for electrorotation. It consists of four identical electrodes that are mutually rotated by $90^{\circ}$. They are made of gold $(500 \mathrm{~nm}$ gold coating deposited on top of a $20 \mathrm{~nm}$ underlying chromium layer) on a glass substrate using a photolithography technology. The object of interest is placed in the area between the four electrodes. Around this workplace, there is a plastic basin of a square shape attached by epoxy glue. Figure 3 depicts the whole chip including the connection pads.

The basin is filled with ethanol and enclosed from above by a glass coverslip to prevent its rapid evaporation and onset of the related fluid flows. This fluidic environment has a density just slightly lower than the density of the microobject. This enables us to keep the micro-object levitating during the experiments in an equilibrium formed by the balance of the downwards directed sedimentation force and the upwards oriented DEP force. Therefore we do not have any friction between the micro-object and the floor of the basin, which would otherwise complicate the modeling task. Furthermore, the low conductivity of ethanol prevents the onset of electrolysis - usually unwanted phenomena occurring during DEP micromanipulation.

As the manipulated micro-object we used a Tetris-shaped piece also shown in Fig. 2A and magnified in Fig. 2B. It is made from SU-8 photoresist and have the dimensions stated in Fig. 2C. We use conical cotton sticks intended for 


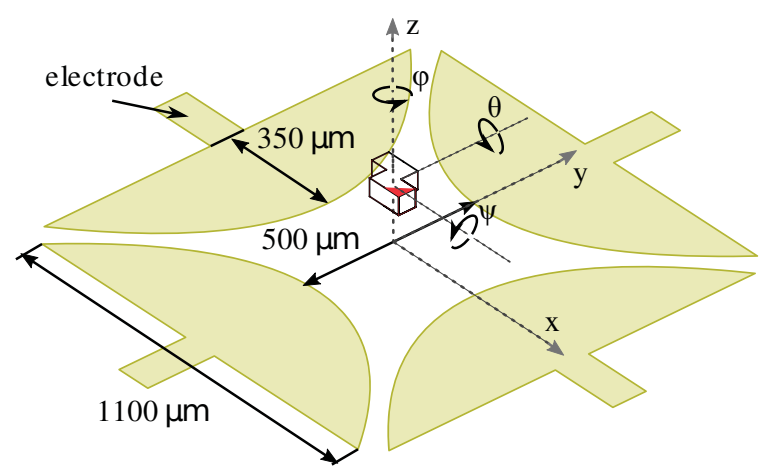

Fig. 4. Dimensions of the electrode array and Tetris-shaped micro-object used in the validation simulations

TABLE I

Properties of the Used MATERials

\begin{tabular}{lll}
\hline \hline Property & Value & Source \\
\hline density of ethanol & $785.22 \mathrm{~kg} \mathrm{~m}^{-3}$ & {$[30]$} \\
viscosity of ethanol & $1.040 \mathrm{mPas}$ at $25^{\circ} \mathrm{C}$ & {$[31]$} \\
relative permittivity of ethanol & 24.3 at $25^{\circ} \mathrm{C}$ & {$[32]$} \\
electrical conductivity of ethanol & $\sim 4 \mu \mathrm{Sm}^{-1}$ & measured $^{*}$ \\
density of SU-8 & $1190 \mathrm{~kg} \mathrm{~m}^{-3}$ & {$[33]$} \\
relative permittivity of SU-8 & 3.2 & {$[34]$} \\
electrical conductivity of SU-8 & $5.556 \times 10^{-11} \mu \mathrm{S} \mathrm{cm}^{-1}$ & {$[34]$} \\
\hline \hline
\end{tabular}

* using multiparameter analyzer Consort C3010

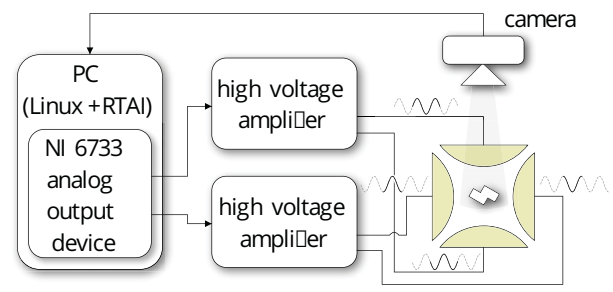

Fig. 5. Scheme of the whole experimental setup.

cleanroom applications to manually arrange the object in place before the experiment starts.

Figure 4 depicts the electrode array including its dimensions and defines the coordinate system used for the description of the micro-objects position and orientation in subsequent sections.

We summarized all the relevant physical properties of the used materials in Table I.

\section{B. Instrumentation}

The glass chip with the electrode array is plugged into a spring connector, and it is placed under an optical tube with an objective $(10 \times)$ attached to its bottom and a camera to its top. The captured video is sent to a regular Linux PC and processed in real-time (we use a custom $\mathrm{C}++$ application taking advantage of the real-time application interface (RTAI) and OpenCV libraries). Although the real-time requirement on image processing is not necessary for analytical purposes, it will come handy when implementing any control algorithm. Since, in the described experimental settings, the object located in the space between the electrodes rotates approximately just around the $z$-axis, we aim at measuring the $x$ and $y$ position of its center and the orientation angle $\phi$ with respect to time. To this purpose, we threshold the captured image, crop it and compute its centroid and the second order central moments. The centroid gives us the planar position and eigenvector associated with the largest eigenvalue of the covariance matrix constructed from these image moments gives us the direction of greatest intensity. For a known shape this can be mapped to its orientation. For symmetrical objects, like the Tetris shape we use, special care has to be taken to solve unambiguity issues. We do so by imposing a constraint that the orientation of the object can not change abruptly.

We generate the harmonic voltage signals using NI 6733 analog output card, then amplify them by a couple of KrohnHite $7600 \mathrm{M}$ high-voltage amplifiers and finally apply them to the electrodes. In the described experiments we used signals with a frequency $f=25 \mathrm{kHz}$. The whole setup is schematically shown in Fig. 5 including an indication of the voltage signals applied to the electrodes in typical ROT experiments $\left(90^{\circ}\right.$ mutually shifted harmonic signals). To validate our theoretical model against the experimental observations, we will also use other values of phase-shifts, and we will also vary the amplitudes between the neighboring electrodes. Note, however, that the signals on opposite electrodes are necessarily always mutually inverted (we use normal and inverted outputs of the amplifiers) and therefore we can set neither the amplitude nor the phase-shift between individual signals independently on all the four electrodes.

\section{EXPERIMENTAL OBSERVATIONS}

In the very first experiment, we placed the Tetris-shaped micro-object close to the center of the electrode array (i.e., the origin of the coordinate system) and applied four harmonic signals shifted in phase by exactly $90^{\circ}$ to the four electrodes it consists from. As a consequence, the Tetris-shaped microobject positions itself in the center of the electrodes and simultaneously moves upwards to a stable equilibrium where it levitates and starts rotating.

The upwards and centering motion is caused by the negative DEP force, which repels the object from high-intensity electric field regions along the edges of the electrodes. With the increasing distance from the electrodes, this force becomes weaker and at some point it is balanced by the sedimentation force. We denote this equilibrium as its levitation height. The described variation of phase on the electrodes creates a rotating electric field inducing the ROT motion of the micro-object.

The experiment revealed that the object rotates around its vertical axis with a nonconstant rotational speed. Figure 6 shows the measurement of the angle $\phi$ and the corresponding angular velocity $\omega=\mathrm{d} \phi / \mathrm{d} t$ obtained by numerical differentiation. Note that we utilized the apparent periodicity of the motion and plotted $\omega$ with respect to $\phi$ rather than time, which will be more suitable for subsequent comparisons against the simulation outcomes. Measurements of the $x$ and $y$ positions of the center of the object presented in Fig. 7 show that apart 

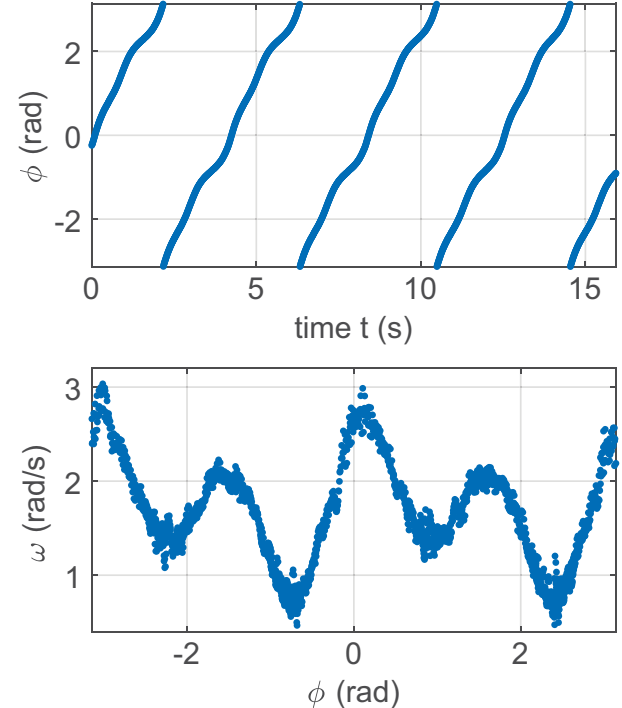

Fig. 6. Experimentally measured rotational behavior of a Tetris-shaped microobject using a standard electrorotational laboratory settings (four electrodes supplied with $90^{\circ}$-shifted harmonic voltages).
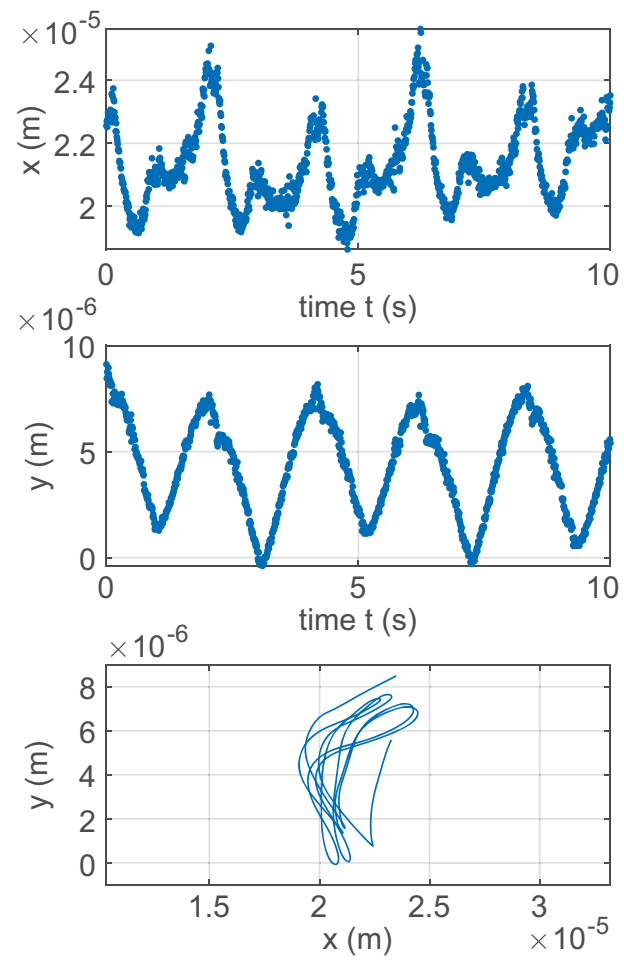

Fig. 7. Experimentally measured $x$ and $y$ positions of the center of a Tetrisshaped micro-object.

from rotation, the object is also subjected to a slight, almost periodic, oscillatory motion in the $x y$-plane. Note also that the rotation did not take place precisely at the center of the electrode array (at the origin of the coordinate system). The reason for this might be a non-symmetry in the design of the experimental chamber or of the paths contacting the individual electrodes.

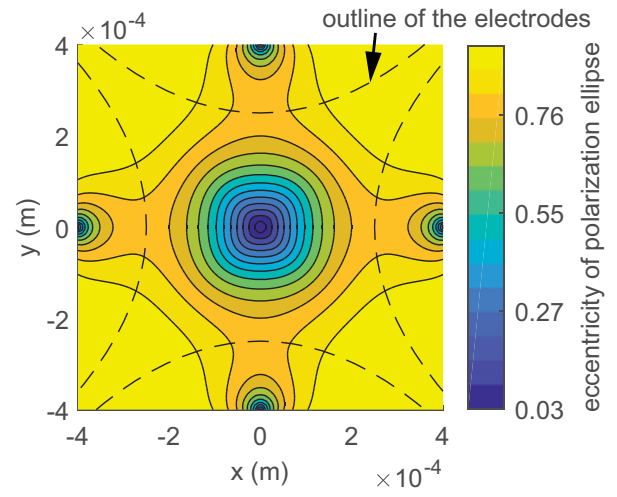

Fig. 8. Eccentricity of the polarizing electric field at $z=136 \mu \mathrm{m}$. Ideally rotating electric field has eccentricity value of zero.

Nonetheless, even if the object's center point laid on the $z$-axis, its rotational speed would not be constant as in the case of a sphere. Although the electric field is ideally rotating along the $z$-axis, anywhere outside this centerline the rotating character of the field deteriorates. This is apparent from Fig. 8, where an eccentricity of the polarizing electric field is plotted at a typical levitation height. Since the field is not precisely rotating in any neighborhood of $z$-axis, let alone in an area that is spanned by the micro-object of interest, the polarization of the object depends on its orientation $\phi$. Therefore also the ROT torque and subsequently the angular velocity $\omega$ varies with $\phi$. This is in sharp contrast with the ROT behavior of a sphere, which achieves due to its geometric symmetry always constant angular velocity. This demonstrates that even in such simple setting the resulting behavior of the micro-object might be non-trivial and every attempt for a precise and accurate control of its motion will necessarily require a suitable controloriented model.

\section{COMPARISON OF MODEL AND EXPERIMENTS}

In this section, we compare the ROT experimental measurements with the proposed control-oriented model of gDEP and hydrodynamics.

To evaluate the model and get the rotational speed of the micro-object, we need to know its position and orientation. As it was already mentioned in section III-B and presented in Figs. 6 and 7, all we can measure is just its $x$ and $y$ coordinates and its orientation $\phi$ around the $z$-axis. From the captured video, we also see that the orientations of the micro-object around the remaining two axes (i.e., angles $\psi$ and $\theta$ ) are zero. The $z$-coordinate of the micro-object (its levitation height) can not be, however, directly measured and has to be at least experimentally estimated. We did so by bringing the object to focus for two situations: while rotating and levitating, and while lying in rest at the bottom floor of the experimental chamber. Subtracting the corresponding readings on the focusing micrometer-screw and adding half of the object's thickness (since it is the position of the center of the object which we are interested in), the levitation height was determined to be $z=136 \mu \mathrm{m}$. Since the measured $x$ and $y$ planar coordinates also evince a periodic behavior as it was 


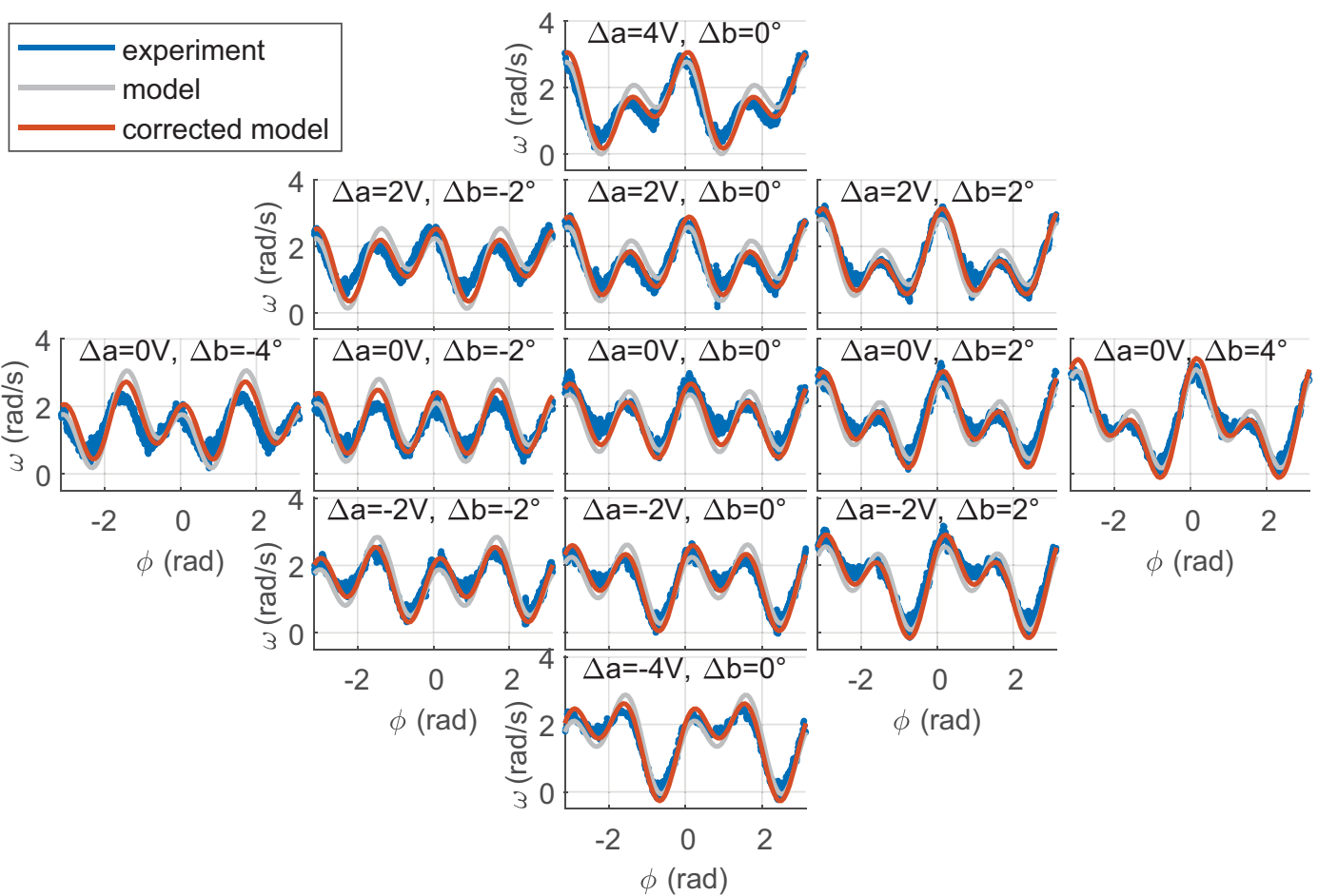

Fig. 9. Dependence of an angular velocity of the micro-object on its orientation for a set of 13 experimental measurements and their comparison with simulation results.

demonstrated in the previous section, we used their averaged value for each specific orientation of the micro-object.

The comparison is made using a set of experiments. In each of them we slightly adjust the voltage signals applied to the electrodes, which results in a different rotational behavior. The voltage waveforms were, starting from the top electrode in Fig. 2 and continuing in a clockwise direction, given by $\left(A-\frac{\Delta a}{2}\right) \cos (2 \pi f t),\left(A+\frac{\Delta a}{2}\right) \cos \left(2 \pi f t-\frac{\pi}{2}+\Delta b \frac{\pi}{180}\right)$, $\left(A-\frac{\Delta a}{2}\right) \cos (2 \pi f t-\pi)$ and $\left(A+\frac{\Delta a}{2}\right) \cos \left(2 \pi f t-\frac{3}{2} \pi+\Delta b \frac{\pi}{180}\right)$, where $A=35 \mathrm{~V}$ is the base amplitude, $t$ represents the time and $\Delta a$ and $\Delta b$ are the parameters differing for each individual experiment. $\Delta a$ basically introduces a difference in the amplitudes of the waveforms between the neighboring electrodes and similarly $\Delta b$ changes their mutual phase-shift from the default $90^{\circ}$. This particular choice of the set of experiments was motivated by the need to stay close enough to the standard ROT settings and prevent the object from escaping from the center of the manipulation area. The resulting rotational behavior is shown in Fig. 9 always with the corresponding $\Delta a$ and $\Delta b$. The individual plots are arranged such that $\Delta a$ increases from the bottom to the top rows and $\Delta b$ increases from the left to the right columns.

Note that for $\Delta a=\Delta b=0$ (all the voltage signals have equal amplitude and successive $90^{\circ}$ phase shift) the rotational velocity should be, because of the symmetry of the field and micro-object, exactly $\frac{\pi}{2}$ periodic. This holds for simulation, but not for the experiment. The model outcomes for such situation better fit the measurements made for $\Delta a=0$ and $\Delta b=2^{\circ}$ and generally that also the other model outcomes better suit the measurements made for $\Delta b$ increased by around $2^{\circ}$. We denote such better fitting model as "corrected model". This indicates that the system is quite sensitive even to small changes of the input parameters. As a consequence, another source of the modeling discrepancies might arise also from any asymmetries in the arrangement of the electrodes (due to asymmetric design of the connection paths or fabrication imprecisions).

\section{SIMULATION ANALYSIS OF OTHER SCENARIOS}

Now when we saw that the proposed model can explain the particular observed experimental behavior, we will use a simulation outcomes to analyze different possible scenarios.

Specifically, we explore how the rotational behavior of the micro-object would change with its varying levitation height. As mentioned above, the levitation height is quite sensitive to even slight variations in the properties of the used materials, mainly their densities. In Fig. 10 we demonstrate this for three different settings of $\Delta b$ and $\Delta a$. Note that with the increasing levitation height the speed of rotation decreases since also the electric field intensity gets weaker. Depending on the particular settings, there is an interesting transitional zone at lower levitation heights (below $50 \mu \mathrm{m}$ ). If the object is levitating near the electrodes, it might not revolve, but it will rather just orient itself with respect to the electrodes by means of EO. The reason for this behavior is that here the electric field is not so nicely rotating as it is in higher levitation heights where it is weaker but smoothed out.

Experimenting with other various shapes of objects and of the electrodes, we further observed, that the spatial (w.r.t. 

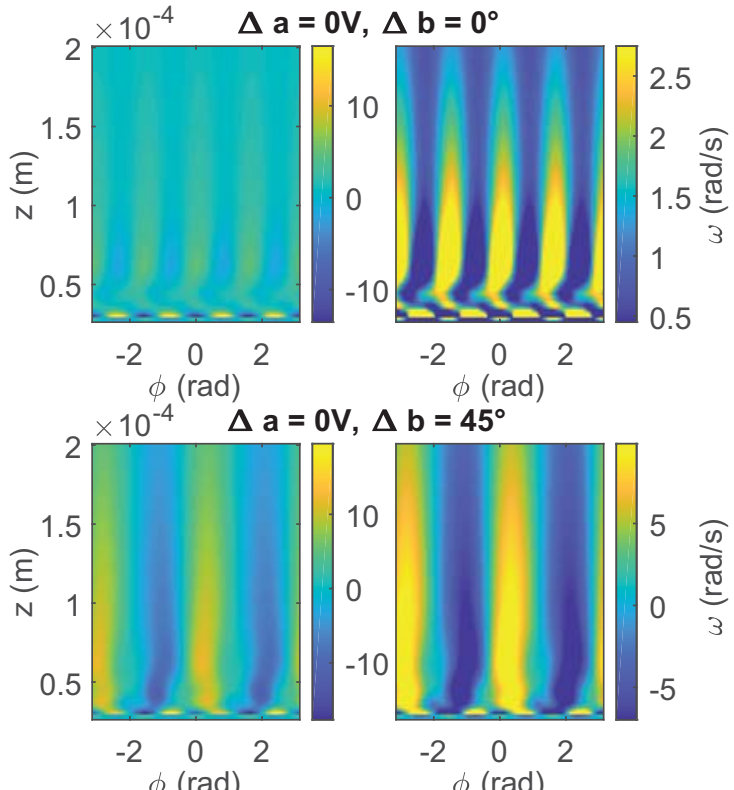

$\mathrm{OV}, \boldsymbol{b}=45^{\circ}$
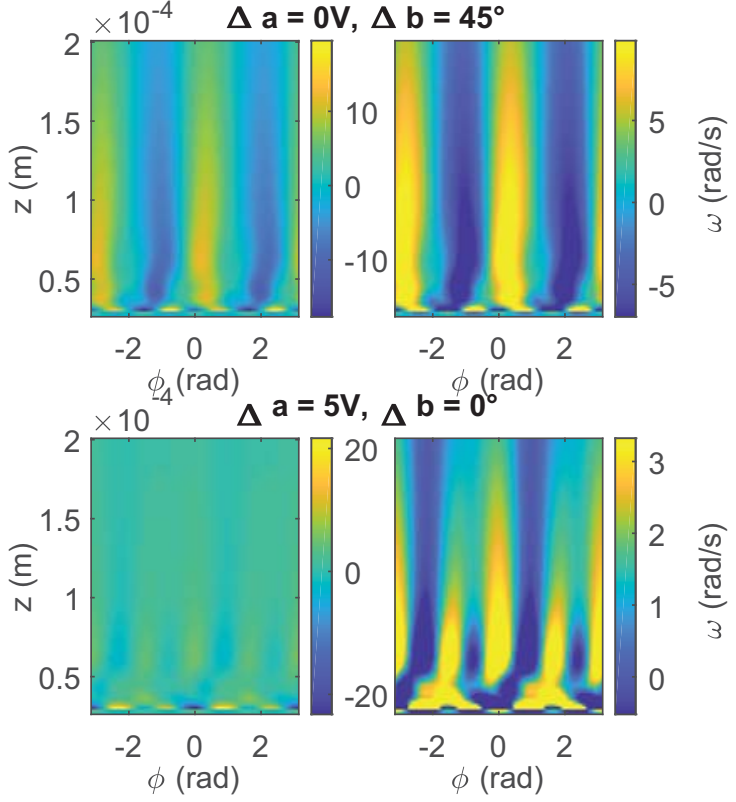

$\begin{array}{lll}-2 & 0 & 2\end{array}$
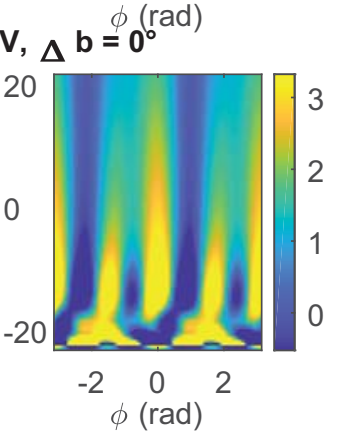

Fig. 10. ROT torque acting on the Tetris-shaped object located on the $z$-axis for varying values of the $z$-coordinate and angle $\phi$. Each row of graphs was generated using different voltages on the electrodes encoded by $\Delta a$ and $\Delta b$ in the respective titles. Two different color scales (unsaturated and saturated) are used for each data set to capture the behavior for lower as well as higher values of $z$.

orientation $\phi$ ) period of angular velocity $\omega$ observed in Figs. 6 and 9 depends on both the spatial periodicity of the source electric field and on the rotational periodicity of the object's shape. Namely, it is equal to their greatest common divisor.

\section{CONCLUSION}

In this paper, we extended the previously developed controloriented model of gDEP [26] for arbitrarily-shaped objects to include also the hydrodynamics. The resulting model can compute the translational and angular velocities of microobjects under gDEP in fractions of a second.

The comparison of the simulation predictions with experimental measurements showed that the model reflects the complex ROT behavior of the Tetris-shaped micro-object.

Since the presented experimental system is very sensitive to even slight changes in the input voltage signals, any control task might be challenging. As a first step towards the simultaneous position and orientation control of micro-objects motivated by the micro-assembly and biology applications, we would like to use the presented model in a controller achieving a steady angular velocity of the arbitrarily-shaped objects.

\section{ACKNOWLEDGMENT}

This work has been supported by the EIPHI Graduate School (contract "ANR-17-EURE-0002"), by the French RENATECH network and its FEMTO-ST technological facility, by the ANR and FNS project CoDiCell (grant No ANR-17CE33-0009), by the MiMedi project funded by BPI France (grant No. DOS0060162/00) and the European Union through the European Regional Development Fund of the Region Bourgogne-Franche-Comte (grant No. FC0013440) and by the Czech Science Foundation within the project P206/12/G014 (Center for advanced bioanalytical technology, http://www. biocentex.cz).

\section{REFERENCES}

[1] H. A. Pohl, Dielectrophoresis: The Behavior of Neutral Matter in Nonuniform Electric Fields. Cambridge ; New York: Cambridge University Press, Dec. 1978.

[2] H. Morgan and N. Green, AC Electrokinetics: Colloids and Nanoparticles, ser. Microtechnologies and microsystems series. Research Studies Press, 2003. [Online]. Available: https://books.google.cz/books?id=FKhPkgEACAAJ

[3] M. Pycraft Hughes, ser. Nano- and Microscience, Engineering, Technology and Medicine. CRC Press, Oct 2002, vol. 4.

[4] T. B. Jones and T. B. Jones, Electromechanics of particles. Cambridge University Press, 2005.

[5] R. Pethig. John Wiley \& Sons, Ltd, Apr 2017.

[6] T. Jones, "Basic theory of dielectrophoresis and electrorotation," IEEE Engineering in Medicine and Biology Magazine, vol. 22, no. 6, pp. 33-42, Nov 2003. [Online]. Available: http://ieeexplore.ieee.org/ document/1304999/

[7] R. Pethig, "Review article-dielectrophoresis: Status of the theory, technology, and applications," Biomicrofluidics, vol. 4, no. 2, p. 022811, Jun 2010. [Online]. Available: http://aip.scitation.org/doi/10. $1063 / 1.3456626$

[8] S.-I. Han, Y.-D. Joo, and K.-H. Han, "An electrorotation technique for measuring the dielectric properties of cells with simultaneous use of negative quadrupolar dielectrophoresis and electrorotation," The Analyst, vol. 138, no. 5, p. 1529, 2013. [Online]. Available: http://xlink.rsc.org/?DOI=c3an36261b

[9] L. Huang, P. Zhao, F. Liang, and W. Wang, Single-cell 3D electrorotation, ser. Methods in Cell Biology. Elsevier, 2018, vol. 148, pp. 97-116. [Online]. Available: https://linkinghub.elsevier.com/retrieve/pii/ S0091679X18300761

[10] G. D. Gasperis, X. Wang, J. Yang, F. F. Becker, and P. R. C. Gascoyne, "Automated electrorotation: dielectric characterization of living cells by real-time motion estimation," Measurement Science and Technology, vol. 9, no. 3, pp. 518-529, Mar 1998. [Online]. Available: http://stacks.iop.org/0957-0233/9/i=3/a=029?key= crossref.61bafdcb1d8b9f96eb7f186589639926

[11] P. Benhal and J. Chase, "System identification and stochastic estimation of dielectric properties of a spherical particle using ac-induced electro-rotation." IEEE, Jun 2015, pp. 332-337. [Online]. Available: http://ieeexplore.ieee.org/document/7169985/

[12] J. Zemánek, T. Michálek, and Z. Hurák, "Phase-shift feedback control for dielectrophoretic micromanipulation," Lab on a Chip, vol. 18, no. 12, pp. 1793-1801, 2018. [Online]. Available: http: //xlink.rsc.org/?DOI=C8LC00113H

[13] J. Zemánek, T. Michálek, and Z. Hurák, "Feedback control for noise-aided parallel micromanipulation of several particles using dielectrophoresis," ELECTROPHORESIS, vol. 36, no. 13, pp. 14511458, 2015. [Online]. Available: https://onlinelibrary.wiley.com/doi/abs/ 10.1002/elps.201400521

[14] M. Kharboutly, A. Melis, M. Gauthier, and N. Chaillet, "2d open loop trajectory control of a micro-object in a dielectrophoresisbased device.” IEEE, Aug 2012, pp. 950-955. [Online]. Available: http://ieeexplore.iee.org/document/6386427/

[15] C. Jiang and J. K. Mills, "Planar cell orientation control system using a rotating electric field," IEEE/ASME Transactions on Mechatronics, vol. 20, no. 5, pp. 2350-2358, Oct 2015. [Online]. Available: http: //ieeexplore.ieee.org/lpdocs/epic03/wrapper.htm?arnumber=6991613 
[16] R. Miller and T. Jones, "Electro-orientation of ellipsoidal erythrocytes. theory and experiment," Biophysical Journal, vol. 64, no. 5, pp. 1588-1595, May 1993. [Online]. Available: https://linkinghub.elsevier. com/retrieve/pii/S0006349593815297

[17] I. Minoura and E. Muto, "Dielectric measurement of individual microtubules using the electroorientation method," Biophysical journal, vol. 90, no. 10, pp. 3739-3748, 2006.

[18] D. Morganti and H. Morgan, "Characterization of non-spherical polymer particles by combined electrorotation and electroorientation," Colloids and Surfaces A Physicochemical and Engineering Aspects, vol. 376, no. 1-3, pp. 67-71, Feb 2011. [Online]. Available: https://linkinghub.elsevier.com/retrieve/pii/S0927775710005479

[19] M. Egger, E. Donath, P. Kuzmin, and V. Pastushenko, "Electrorotation of dumb-bell shaped particles," Bioelectrochemistry and Bioenergetics, vol. 26, no. 3, pp. 383-393, Dec 1991. [Online]. Available: http://linkinghub.elsevier.com/retrieve/pii/030245989185001I

[20] J. J. Arcenegui, P. García-Sánchez, H. Morgan, and A. Ramos, "Electro-orientation and electrorotation of metal nanowires," Physical Review E, vol. 88, no. 6, Dec 2013. [Online]. Available: https: //link.aps.org/doi/10.1103/PhysRevE.88.063018

[21] H. Morgan and N. G. Green, "Dielectrophoretic manipulation of rod-shaped viral particles," Journal of Electrostatics, vol. 42, no. 3, pp. 279-293, Dec 1997. [Online]. Available: http://linkinghub.elsevier.com/ retrieve/pii/S0304388697001599

[22] Y. Liu, W. K. Liu, T. Belytschko, N. Patankar, A. C. To, A. Kopacz, and J.-H. Chung, "Immersed electrokinetic finite element method," International Journal for Numerical Methods in Engineering, vol. 71, no. 4, pp. 379-405, Jul 2007. [Online]. Available: http://doi.wiley.com/10.1002/nme.1941

[23] A. di Biasio, L. Ambrosone, and C. Cametti, "Numerical simulation of dielectric spectra of aqueous suspensions of nonspheroidal differently shaped biological cells," Journal of Physics $D$ Applied Physics, vol. 42, no. 2, p. 025401, Jan 2009 [Online]. Available: http://stacks.iop.org/0022-3727/42/i=2/a=025401? $\mathrm{key}=$ crossref.daf9f722476a5030f1b7e54b61b2d487

[24] P. P. Mathai, A. J. Berglund, J. Alexander Liddle, and B. A. Shapiro, "Simultaneous positioning and orientation of a single nano-object by flow control: theory and simulations" New Journal of Physics, vol. 13, no. 1, p. 013027, Jan 2011 [Online]. Available: http://stacks.iop.org/1367-2630/13/i=1/a=013027? key=crossref.ed3f71e39519a078c4e5fd35d000a754

[25] J. Happel and H. Brenner, Low Reynolds number hydrodynamics: with special applications to particulate media. Prentice-Hall, 1965, googleBooks-ID: 4MpAQAAMAAJ.

[26] T. Michálek, A. Bolopion, Z. Hurák, and M. Gauthier, "Control-oriented model of dielectrophoresis and electrorotation for arbitrarily shaped objects," Physical Review E, 2019, (accepted for publication).

[27] P. Wong, T.-H. Wang, J. Deval, and C.-M. Ho, "Electrokinetics in micro devices for biotechnology applications," IEEE/ASME Transactions on Mechatronics, vol. 9, no. 2, pp. 366-376, Jun 2004. [Online]. Available: http://ieeexplore.iee.org/document/1306450/

[28] J. J. Abbott, K. E. Peyer, M. C. Lagomarsino, L. Zhang, L. Dong, I. K. Kaliakatsos, and B. J. Nelson, "How should microrobots swim?" The International Journal of Robotics Research, vol. 28, no. 11-12, pp. 1434-1447, Nov 2009. [Online]. Available: http://journals.sagepub.com/doi/10.1177/0278364909341658

[29] S. Klumpp, C. T. Lefèvre, M. Bennet, and D. Faivre, "Swimming with magnets: From biological organisms to synthetic devices," Physics Reports, vol. 789, pp. 1-54, Jan 2019. [Online]. Available: https://linkinghub.elsevier.com/retrieve/pii/S0370157318302862

[30] J. A. Dean, Lange's Handbook of ChemistryHardcover, 15th ed. McGraw-Hill Professional, 1998. [Online]. Available: https://trove.nla gov.au/work/9499973

[31] "Pure Component Properties." [Online]. Available: https://www.cheric org/research/kdb/hcprop/cmpsrch.php

[32] "Dielectric Constants of common Liquids." [Online]. Available: https: //www.engineeringtoolbox.com/liquid-dielectric-constants-d_1263.html

[33] "SU-8 photoresist." [Online]. Available: http://www.mit.edu/ 6.777/ matprops/su-8.htm

[34] "MicroChem." [Online]. Available: http://microchem.com/pdf/ SU-8-table-of-properties.pdf

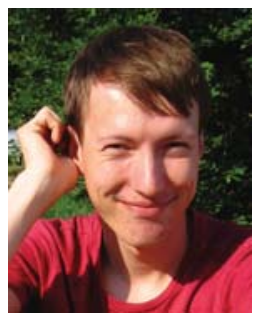

Tomáš Michálek received his Ing. ( M.Sc.) degree in Cybernetics and Robotics from the Czech Technical University in Prague, Prague, Czech Republic, in 2015. He is currently working toward the Ph.D. degree in Control Engineering and Robotics.

His research interests include mathematical modeling and control design for noncontact micromanipulation via dielectrophoresis and electrorotation.

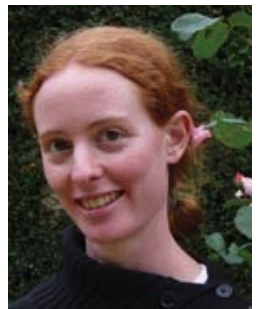

Aude Bolopion received her Ph.D. degree in robotics in 2010 from the University of Pierre et Marie Curie, Paris, France.

She is currently a CNRS researcher at the FEMTO-ST Institute, Besançon, France. She has been a member of the Biomedical Micro Nano Robotics team since 2011. From 2007 and 2011 she was a member of the ISIR micromanipulation team. Her research interests are focused on teleoperation and haptic feedback at the nanoscale, and on noncontact micromanipulation.

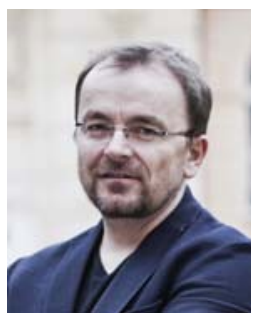

Zdeněk Hurák received the Ing. ( M.Sc.) degree in aerospace electrical engineering (summa cum laude) from Military Academy, Brno, Czech Republic, in 1997. He received the Ph.D. degree in cybernetics and robotics from the Czech Technical University, Prague, Czech Republic, in 2004. Currently he is an associate professor of control engineering at Faculty of Electrical Engineering, Czech Technical University in Prague, Czech Republic. His research focus is on computational methods for optimal, robust and distributed control and their applications in electromechanical systems, including non-contact (micro)manipulation using dielectrophoresis and magnetophoresis, more info on his group webpage http://aa4cc.dce.fel.cvut.cz.

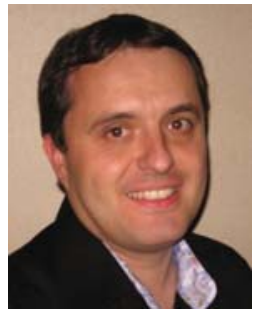

Michaël Gauthier received his $\mathrm{PhD}$ degree in Automation and Robotics from the Université de Franche Comté, France in 2002.

$\mathrm{He}$ is currently CNRS Senior scientist research at FEMTO-ST institute and the vice-head of FEMTOST. He has been granted by the French CNRS bronze medal (Early Career Award) in 2011. He is author or co-author of more than 28 journal papers and 42 conference papers in the field of micro-nano-robotics His research interests are focused on microworld modeling, micro and nano manipulation, dexterous microhandling and high speed control of non-contact micromanipulations. 